\title{
Detection of Spinal Muscular Atrophy Using a Duplexed Real-Time PCR Approach With Locked Nucleic Acid-Modified Primers
}

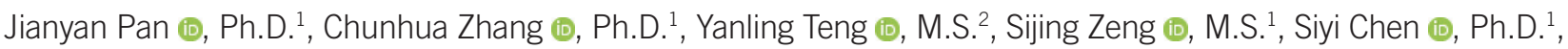 \\ Desheng Liang (i), M.D., Ph.D. ${ }^{1}$, Zhuo Li $\mathbb{C}^{0}$, Ph.D. ${ }^{1}$, and Lingqian Wu (i), M.D., Ph.D. ${ }^{1}$ \\ ${ }^{1}$ Center for Medical Genetics \& Hunan Key Laboratory of Medical Genetics, School of Life Sciences, Central South University, Changsha, Hunan, China; \\ ${ }^{2}$ Laboratory of Molecular Genetics, Hunan Jiahui Genetics Hospital, Changsha, Hunan, China
}

Background: Spinal muscular atrophy (SMA) is an autosomal recessive neuromuscular disorder mainly caused by homozygous deletions that include exon 7 of the survival motor neuron 1 (SMN1) gene. A nearby paralog gene, SMN2, obstructs the specific detection of SMN1. We optimized a duplexed real-time PCR approach using locked nucleic acid (LNA)modified primers to specifically detect SMN1.

Methods: An LNA-modified primer pair with 3' ends targeting SMN1 specific sites c.835$44 \mathrm{~g}$ and C.840C was designed, and its specificity was examined by real-time PCR and Sanger Sequencing. A duplexed real-time PCR approach for amplifying SMN1 and control gene albumin $(A L B)$ was developed. A randomized double-blind trial with 97 fresh peripheral blood samples and 25 dried blood spots (DBS) was conducted to evaluate the clinical efficacy of the duplexed approach. This new approach was then used to screen 753 newborn DBS.

Results: The LNA-modified primers exhibited enhanced specificity and $6.8 \%$ increased efficiency for SMN1 amplification, compared with conventional primers. After stabilizing the SMN1 test by optimizing the duplexed real-time PCR approach, a clinical trial validated that the sensitivity and specificity of our new approach for detecting SMA patients and carriers was $100 \%$. Using this new approach, 15 of the screened 753 newborns were identified as carriers via DBS, while the rest were identified as normal individuals. These data reveal a carrier rate of $1.99 \%$ in Hunan province, South Central China.

Conclusions: We have developed a novel, specific SMN1 detection approach utilizing real-time PCR with LNA-modified primers, which could be applied to both prenatal carrier and newborn screening.

Key Words: Spinal muscular atrophy, Survival motor neuron 1, Locked nucleic acid, Realtime PCR, Dried blood spot, Newborn screening
Received: January 21, 2020

Revision received: March 24, 2020

Accepted: July 17, 2020

Corresponding author: Zhuo Li, Ph.D. Center for Medical Genetics \& Hunan Key Laboratory of Medical Genetics, School of Life Sciences, Central South University, Changsha, Hunan 410078, China

Tel: +86-731-84805359

E-mail: lizhuo@sklmg.edu.cn

\section{Co-corresponding author:}

Lingqian Wu, M.D., Ph.D.

Center for Medical Genetics \& Hunan Key Laboratory of Medical Genetics, School of Life Sciences, Central South University, Changsha, Hunan 410078, China Tel: +86-731-84805252

E-mail: wulingqian@sklmg.edu.cn

\section{C) (1)}

() Korean Society for Laboratory Medicine

This is an Open Access article distributed under the terms of the Creative Commons Attribution Non-Commercial License (https://creativecommons.org/licenses/by-nc/4.0) which permits unrestricted non-commercial use, distribution, and reproduction in any medium, provided the original work is properly cited.

\section{INTRODUCTION}

Spinal muscular atrophy (SMA) is the most common autosomal recessive neurodegenerative disorder resulting from loss of motor neurons in the spinal cord and brainstem, leading to hypoto- nia, progressive proximal muscle weakness, and respiratory distress. SMA has a reported incidence of approximately 1 in 7,000 live births [1] and a high carrier rate ranging from $1.38 \%$ to $2.13 \%[2,3]$. This severe neuromuscular disease has been recommended for routine carrier screening in the general popula- 
tion by the American College of Medical Genetics [4, 5].

Approximately $95 \%$ of SMA patients show a homozygous absence of exon 7 or both exons 7 and 8 of the survival of motor neuron 1 (SMN1) gene, which is located on chromosome $5 q 13$ [6]. This region contains two nearly identical copies: the telomeric copy (the SMN1 gene) and the centromeric copy (the SMN2 gene), which increases the difficulty of molecular diagnosis of SMA by detecting SMN1 exon 7 copy number. The SMN1 gene differs from the SMN2 gene by only five single nucleotides including four in the non-coding region and one in the coding region (exon 7) [7]. The only coding region variant, a single Cto-T substitution between SMN1 and SMN2 in exon 7 (c.840C > T), results in a decrease in full-length $S M N 2$ transcripts and is considered to mainly differentiate SMN1 from SMN2.

Real-time PCR and PCR-denaturing high performance liquid chromatography (PCR-DHPLC) are the most common technologies for SMA large-scale population screening [8-10]. However, the primers or probes used in traditional SMA PCR strategies, which cover the single differential base pair, may not fully ensure SMN1 amplification specificity. Recent studies have shown that oligonucleotide sequences modified by a locked nucleic acid (LNA), an available alternative nucleic acid that contains a methylene bridge connecting the 2 -oxygen and $4{ }^{\prime}$-carbon in the ribose moiety, display enhanced specificity, sensitivity, and hybridization stability toward complementary DNA and RNA [11, 12]. Substitution with an LNA monomer increases primer melting temperature $(\mathrm{Tm})$ values from +2 to $+8^{\circ} \mathrm{C}$ for DNA, which may be related to the increased thermal stability of the base pairs. Additionally, the increase in Tm value depends on the position and number of the LNAs in oligonucleotide chain [11].

Therefore, to further optimize a real-time PCR approach for SMN1 detection, we modified conventional primers and probes with LNAs and validated the test performance using clinical samples. We also expanded the application of this new technology for SMA screening in newborns using dried blood spots (DBS). To the best of our knowledge, this is the first duplexed real-time PCR approach developed using LNA-modified primers for detecting the copy number of SMN1 exon 7.

\section{MATERIALS AND METHODS}

\section{Sample sources}

In this prospective study, 157 peripheral blood samples (40 patients, 74 carriers, and 43 normal individuals), whose genotypes were confirmed by multiplex ligation-dependent probe amplification (MLPA) at Hunan Jiahui Genetics Hospital, Hunan, China, from May 2014 to December 2018, were collected. Of these, 60 samples were used to establish the real-time PCR test, while 97 samples (20 patients, 54 carriers, and 23 normal individuals) and another 25 DBS (derived from peripheral blood) with genotypes verified using MLPA were randomly assigned in a double-blind design. Genomic DNA was extracted from peripheral blood cells using the QuickGene DNA whole blood kit $L$ and the QuickGene-610L automated system (FUJIFILM Corporation, Tokyo, Japan) and kept at $-20^{\circ} \mathrm{C}$. Since 2018,753 newborn DBS samples from the Han population preserved at $-20^{\circ} \mathrm{C}$ have been randomly selected from the Maternal and Child Health Hospital of Hunan Province, China, for SMA screening. Genomic DNA was extracted from the DBS using the Nucleic Acid Extraction Reagent (Shenzhen Uni-medica Co., Ltd., Shenzhen, China). Our study complies with the Declaration of Helsinki and was approved by the Institutional Review Board of the Center for Medical Genetics, Central South University, Hunan (201503002). Informed consent was obtained from individuals and parents of the newborns involved in the study.

\section{Primers and probes}

The primers and probes were designed using Primer Premier 5 (Premier Biosoft, San Francisco, CA, USA). The 3' ends of the specific forward primer (SMN1-F) and reverse primer (SMN1-R) used for amplifying the SMN1 gene (NM_000344.3) were designed to exactly target the c.835-44g and c.840C sites, respectively, and were modified by LNAs (Fig. 1). To further prevent nonspecific amplification of the SMN2 gene (NM_017411.3), we also designed a blocked probe (SMN2-B) targeting the c.840T site; the 18th and 19th bases of this probe were modified with an LNA, and the 3' end was modified with a C3 spacer. The primers for the control gene, albumin (ALB; NM_000477.7), were designed in the region between intron 11 and exon 12. Reporter dyes 6-carboxyfluorescein (FAM) and cyanine 5 (Cy5) were used to label the TaqMan probes of SMN1 and $A L B$, respectively. To further optimize the amplification specificity for SMN1, we designed two primer pairs: nonspecific primers SMN-F/R and specific primers SMN1-F1/R1 without LNA modification. All primers and probes (Supplemental Data Table S1) were synthesized by Sangon Biotech (Shanghai, China). Sanger Sequencing with different primer combinations was also conducted by Sangon Biotech (Fig. 1).

\section{MLPA}

A total of 157 peripheral blood samples were analyzed using MLPA to confirm the copy number of exon 7 and 8 in the SMN1 


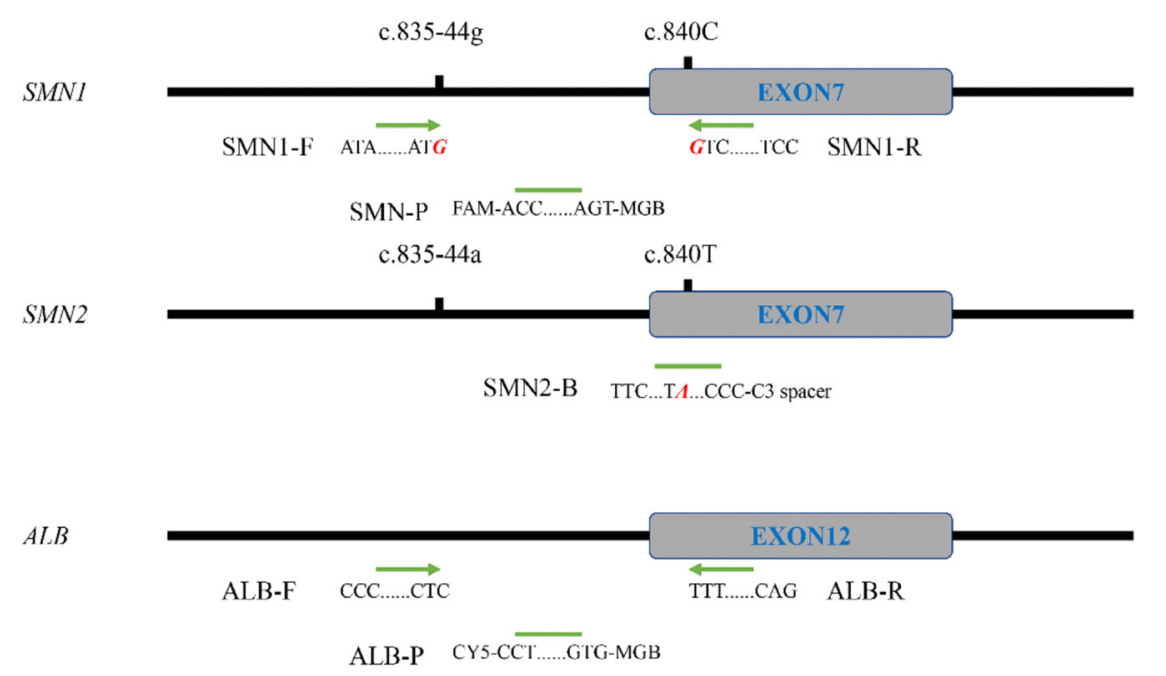

Fig. 1. Diagram of the binding location of the SMN1, SMN2, and ALB primers and probes. In primers SMN1-F/R, the 3' end of the forward primer and reverse primer, targeting the c.835-44g and c.840C site, respectively, has been LNA-modified; the modified bases are indicated in red.

Abbreviations: SMN, survival motor neuron; $A L B$, albumin; LNA, locked nucleic acid; F, forward; $\mathrm{R}$, reverse; $\mathrm{P}$, probe.

and SMN2 genes using the SALSA MLPA P021 SMA kit (MRCHolland, Amsterdam, The Netherlands).

\section{Real-time PCR}

Real-time PCR was performed using an ASA-9600 Real-Time PCR System (Suzhou Baiyuan Gene Technology Co., Ltd., Suzhou, China) with a $2 \times$ AceQ U+ Probe Master MIX (Vazyme Biotech Co., Ltd., Nanjing, China) in triplicate and run according to the manufacturer's protocol. We initially used a temperature gradient (from $54-60^{\circ} \mathrm{C}$ ) to determine the optimal PCR annealing temperature for maximal and stable amplification efficiency. PCR was then performed with the following amplification conditions: $45^{\circ} \mathrm{C}$ for 2 minutes and $95^{\circ} \mathrm{C}$ for 10 minutes, followed by 45 cycles of $95^{\circ} \mathrm{C}$ for 15 seconds and $60^{\circ} \mathrm{C}$ for 40 seconds. Partial DNA sequences of $S M N 1$ and $A L B$ were cloned into a single pUC57 vector as the standard samples for quantifying SMN1 and ALB copy number (Supplemental Data Fig. S1). To facilitate test interpretation, we defined three rate value $(R)$ ranges according to the relative rate between the copy number of SMN1 and $A L B$ after one duplexed reaction. Based on the previous experiment with 60 samples, patients, carriers, and normal individuals were assigned ratios of $0-0.1,0.35-0.65$, and $0.8-1.2$, respectively. To validate clinical efficacy, another 97 clinical DNA samples extracted from peripheral blood were recruited and tested by randomized double-blind design using the established du- plexed real-time PCR approach.

\section{RESULTS}

Primer specificity was increased by LNA modification

As the homozygous deletion of SMN1 exon 7 in the patient DNA was previously confirmed, increased PCR products would indicate increased nonspecific amplification of pseudogene SMN2. Accordingly, the real-time PCR results showed that the internal control $A L B$ amplification and targeted gene amplification with complete nonspecific primers SMN-F/R entered the exponential phase at the same time, indicating complete nonspecific SMN2 amplification. When the forward or reverse primer was replaced with the allele-specific primer SMN1-F1 (c.835-44g) or SMN1R1 (c.840C), respectively, the exponential phase was obviously delayed, suggesting less SMN2 amplification. Thus, although both allele-specific primers exhibited high specificity for SMN1, they could not eliminate the nonspecific amplification of SMN2. When the allele-specific primers were further modified with LNAs, no SMN2 amplification was detected with either a single LNAmodified primer or both LNA-modified primers (SMN1-F/R); the amplification curves did not form or remained under the threshold line in valid cycles, implying an extreme enhancement of SMN1 specificity following LNA modification (Fig. 2). A similar increase in specificity due to LNA modification was observed in 


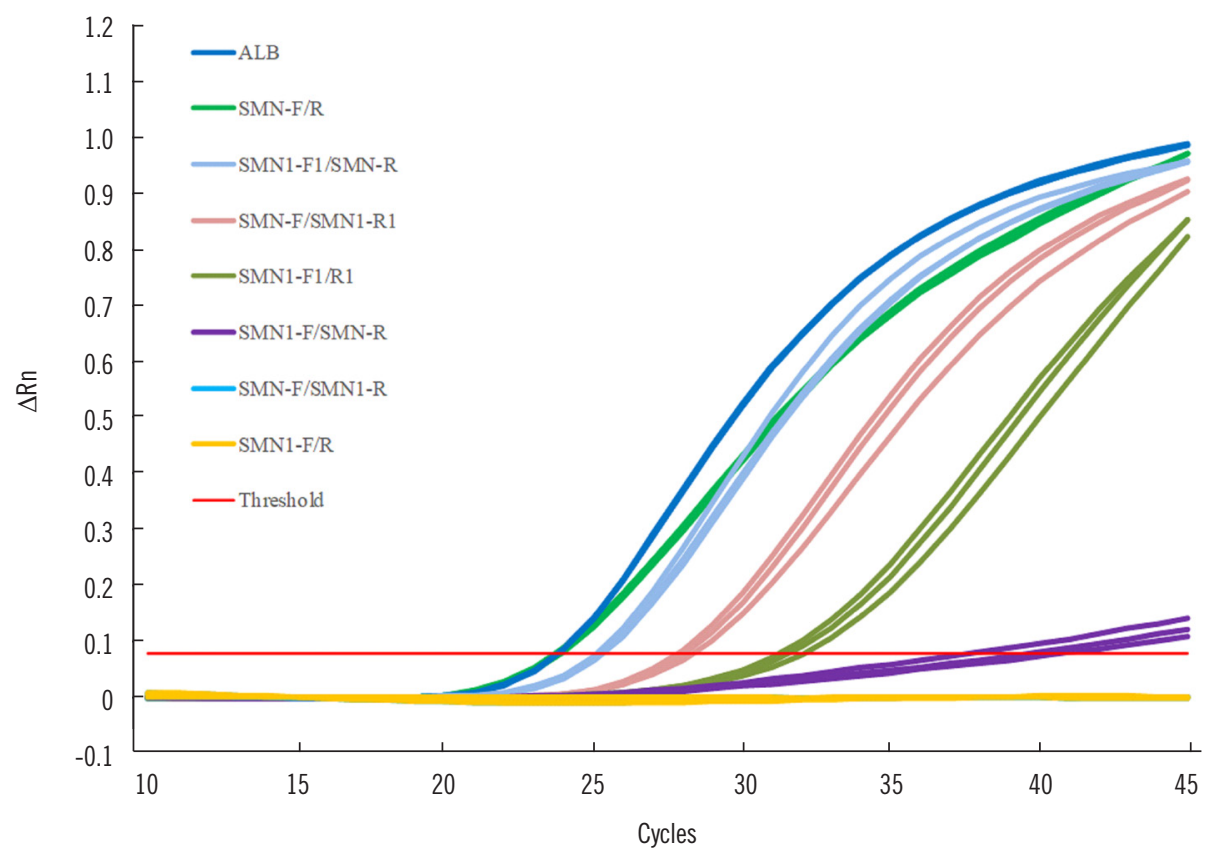

Fig. 2. The amplification specificity of seven different SMN1 primer groups detected by real-time PCR using SMA patient DNA as the template. Each group was set up in triplicate.

Abbreviations: SMN1, survival motor neuron 1; $A L B$, albumin; SMA, spinal muscular atrophy; $\triangle \mathrm{Rn}$, normalized reporter; F, forward; R, reverse.

the Sanger sequencing chromatograms of the two SMN1 and SMN2 differential sites (Supplemental Data Fig. S2).

\section{Validation using clinical peripheral blood and DBS samples} The results revealed a uniform efficiency of approximately $95 \%$ throughout the temperature range $\left(54-60^{\circ} \mathrm{C}\right)$ when using normal individual DNA samples with the SMN1-F/R primers, which was $6.8 \%$ higher than when primers SMN1-F1/R1 (without LNA modification) were used at $60^{\circ} \mathrm{C}$ (Fig. $3 \mathrm{~A} \& 3 \mathrm{~B}$ ). Hence, LNA modification may not only improve primer specificity but also promote amplification efficiency. The three genotypes are clearly identifiable on the basis of the amplification curve. Normal individual samples showed a synchronous cycle threshold (Ct) with $A L B$, indicating two copies of SMN1 exon 7 (Fig. $3 C$ ), while the carrier samples displayed a $1 \mathrm{Ct}$ extension compared with $A L B$, suggesting one copy of SMN1 exon 7 (Fig. 3D). No amplification of SMN1 was observed in SMA patient samples, confirming homozygous deletion of SMN1 exon 7 and no detectable crossreactivity with SMN2 (Fig. 3E). These 60 test results demonstrated $100 \%$ concordance with the corresponding clinical status. $A L B$ demonstrated stable amplification in all samples in the duplexed test with nearly the same Ct (Fig. 3).

Of the 97 samples, 20 were considered to be SMA patients (average R: 0, R range: $0-0$ ), 54 were classified as SMA carriers (average R: $0.56 \pm 0.03, \mathrm{R}$ range: $0.49-0.62$ ), and the remaining 23 samples were classified as normal individuals (average $R$ : $1 \pm 0.09$, $\mathrm{R}$ range: $0.8-1.18$ ). After unblinding the trial, all the test results were completely consistent with their original phenotypes and genotypes, which were confirmed by MLPA. Therefore, the sensitivity and specificity of our new real-time PCR technology was $100 \%$ for SMA patients with no copies of SMN1, carriers with one copy of $S M N 1$, and normal individuals with two copies of SMN1 (Table 1).

To expand the application of this new approach to newborn screening for SMA, we also performed a double-blind test using 25 DBS samples (5 SMA patients, 10 SMA carriers, and 10 normal individuals). The SMN1 categorical genotypes obtained from the test also showed 100\% concordance with the clinical status of the samples (Table 2).

Newborn screening for SMA patients and carriers using the duplexed approach with DBS

Of 753 newborn DBS samples, 15 newborns were identified as SMA carriers with one copy of SMN1 (average R: $0.54 \pm 0.06$, R range: 0.43-0.63) and 729 were identified as normal individuals with two copies of $S M N 1$ (average $R: 0.97 \pm 0.12$, $R$ range: 0.8-1.2). Surprisingly, nine newborns were identified as having three copies of SMN1, with an R-value of approximately 1.5 (av- 


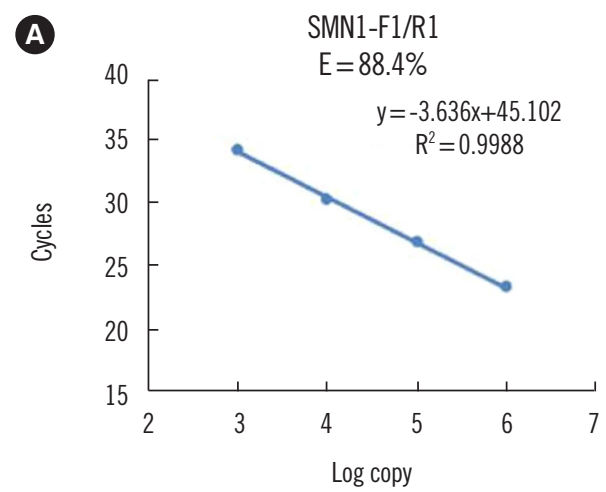

C

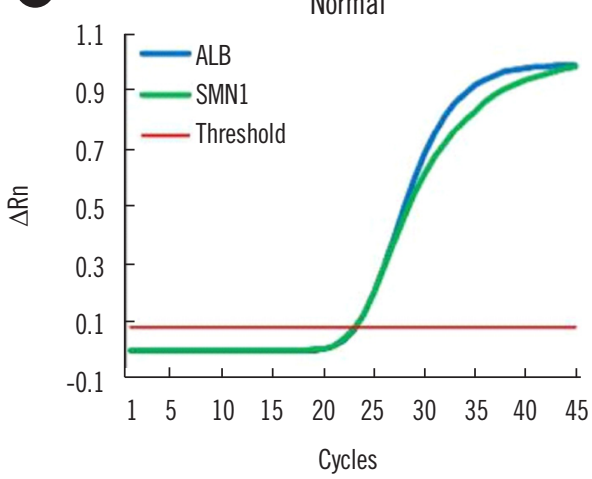

(D)

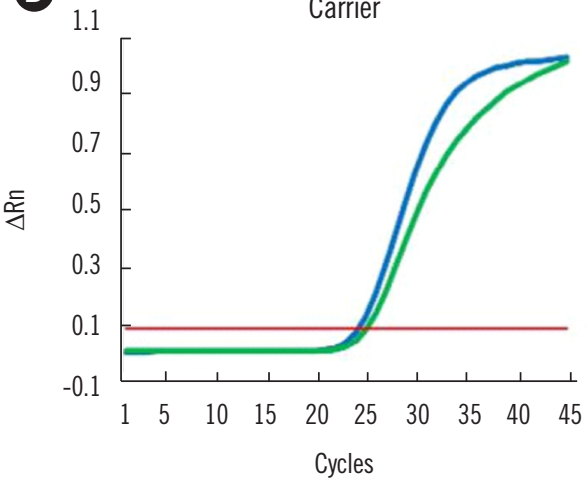

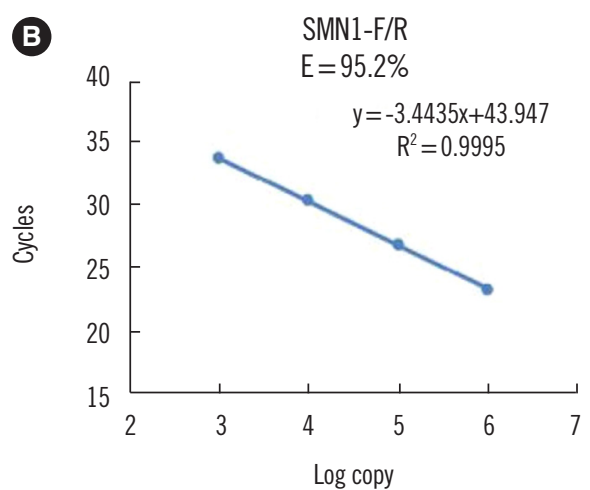

E
Patient

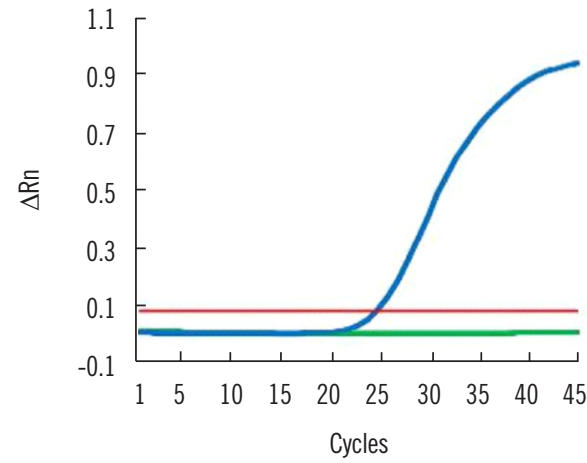

Fig. 3. Primer amplification efficiency and real-time PCR amplification curves. (A) The amplification efficiency of primers SMN1-F1/R1 was $88.4 \%$ at $60^{\circ} \mathrm{C}$. (B) The amplification efficiency of primers SMN1-F/R was $95.2 \%$ at $60^{\circ} \mathrm{C}$. (C) The SMN1 and $A L B$ amplification curves in normal individual samples overlapped at the threshold. (D) In SMA carrier samples, the SMN1 amplification curve exhibited an approximate one cycle delay compared with the $A L B$ curve at the threshold. (E) In SMA patient samples, only the $A L B$ gene was amplified.

Abbreviations: SMN1, survival motor neuron 1; $A L B$, albumin; SMA, spinal muscular atrophy; F, forward; R, reverse; $\triangle$ Rn, normalized reporter.

Table 1. Validation using 97 peripheral blood samples by real-time PCR

\begin{tabular}{cccccc}
\hline & \multicolumn{5}{c}{ SMN1 copy number } \\
\cline { 2 - 6 } & 0 & 1 & 2 & 3 & Total \\
\hline Individuals & 20 & 54 & 23 & 0 & 97 \\
R-value* & 0 & $0.56 \pm 0.03$ & $1 \pm 0.09$ & - & - \\
Sensitivity & $100 \%$ & $100 \%$ & $100 \%$ & & \\
$95 \% \mathrm{Cl}$ & $80-100 \%$ & $92-100 \%$ & $82-100 \%$ & - & - \\
Specificity & $100 \%$ & $100 \%$ & $100 \%$ & & \\
$95 \% \mathrm{Cl}$ & $94-100 \%$ & $90-100 \%$ & $94-100 \%$ & - & - \\
\hline
\end{tabular}

${ }^{*} \mathrm{R}$-value $($ mean $\pm \mathrm{SD})$ indicates the relative rate between the copy number of SMN1 and $A L B$.

Abbreviations: SMN1, survival motor neuron $1 ; A L B$, albumin; R, rate value; $\mathrm{Cl}$, confidence interval.

erage R: $1.49 \pm 0.08, \mathrm{R}$ range: $1.38-1.61)$. These data demonstrate, for the first time, a carrier rate of $1.99 \%$ (95\% confidence interval, 1.12-3.26\%) in Hunan Province, South Central China (Table 2).
Table 2. Detection of 25 DBS derived from peripheral blood and screening of 753 newborn DBS samples using real-time PCR

\begin{tabular}{llccccc}
\hline & \multicolumn{5}{c}{ SMN1 copy number } \\
\cline { 3 - 7 } & & 0 & 1 & 2 & 3 & Total \\
\hline \multirow{2}{*}{$\begin{array}{l}\text { DBS from } \\
\text { peripheral blood }\end{array}$} & Individuals & 5 & 10 & 10 & 0 & 25 \\
R-value & 0 & $0.51 \pm 0.05$ & $1.01 \pm 0.06$ & - & - \\
DBS from & Individuals & 0 & 15 & 729 & 9 & 753 \\
newborn & R-value & - & $0.54 \pm 0.06$ & $0.97 \pm 0.12$ & $1.49 \pm 0.08$ & - \\
& Rate & 0 & $1.99 \%$ & $96.81 \%$ & $1.20 \%$ & $100 \%$ \\
\hline
\end{tabular}

Abbreviations: SMN1, survival motor neuron 1; $\mathrm{R}$, rate value; DBS, dried blood spots.

\section{DISCUSSION}

The high carrier rate and severe clinical outcome of infantile SMA necessitates performing timely carrier screening for pregnant women and couples with a birth plan [13, 14]. In addition, as two US Food and Drug Administration approved drugs for SMA treatment have entered clinical practice [15], newborn 
screening for SMA by genetic testing would be an effective measure for maximizing the benefits of presymptomatic intervention and guide subsequent healthcare.

Although several technologies, such as MLPA, droplet digital $\mathrm{PCR}$, and next generation sequencing (NGS)-based approach, display high specificity and sensitivity for SMA detection [16,17], relatively complex processes, equipment requirements, and high costs currently obstruct their application in population screening, especially for grass-root units. PCR-based approaches remain preferable options for large-scale population screening. The main challenge of conventional PCR-related approach in detecting SMN1 absence is the potential nonspecific amplification of SMN2, owing to the nearly identical sequence of SMN1 and SMN2.

In accordance with the allele-specific PCR principle, when we performed the real-time PCR approach using SMA patients' DNA with conventional PCR primers, nonspecific amplicons of SMN2 were detected, indicating that conventional real-time PCR may have a potential risk of producing false-negative signals. To reduce this risk, we introduced LNA modification in the primers to further promote the specificity of SMN1 amplification; LNA oligonucleotides increase primer or probe binding strength for complementary sequences, thereby enhancing test specificity and enabling detection of single nucleotide mismatches [18, 19]. A previous SMA detection study reported that a five LNA monomer-modified probe, targeting the differential site c.888+100a in the SMN1 noncoding region, markedly increased detection specificity. When the annealing temperature raised to $65^{\circ} \mathrm{C}$, no nonspecific amplicons were detected using the LNA probe in SMA patients [20]. However, that technology was not sufficiently precise for reliable carrier identification. Additionally, another study using a similar probe targeting c.888+100a, reported eight false-positive cases [21], which may also cause false-negative results owing to the conversion of SMN1 into SMN2 following a c.840C > T substitution. We utilized two differential sites in SMN1, c.835-44g and c.840C, at the $3^{\prime}$ end of our designed primers. Additionally, in contrast to the five LNA monomer-modified probe, we used only one LNA to modify the $3^{\prime}$ end of the designed primers, consequently obtaining a significant increase in specificity.

Furthermore, LNA-modified primers appear to enhance the stability and sensitivity of SMN1 detection in a duplexed test. Our results revealed that although the duplexed test contained complex components, including four primers and three probes, as well as different templates, stable and satisfactory amplification efficiency was maintained even when the annealing temperature was changed from 54 to $60^{\circ} \mathrm{C}$. Subsequent clinical ef- ficacy demonstrated $100 \%$ concordance with each group of patients, carriers, and normal individuals.

A cohort of 753 newborn DBS samples screened using our novel duplexed real-time PCR approach, demonstrated, for the first time, a carrier rate of $1.99 \%$ among the Han population of Hunan Province. This result is similar to the carrier rates of $1.90 \%$ and $1.83 \%$ revealed by studies conducted in East China [22] and Korea [23], respectively.

The cost for our new approach is approximately $\$ 1$ dollar per test, and the results are available within three hours. Although approximately $95 \%$ of SMA patients with a homozygous absence of exon 7 and corresponding carriers can be detected using this economical and rapid test, a small number of patients with SMA caused by single nucleotide variation and $(2+0)$ carriers (with two copies of SMN1 on the same chromosome) cannot be identified [24, 25]. Further efforts should focus on the development of more comprehensive screening approaches for SMA.

In conclusion, we have developed a duplexed real-time PCR approach using LNA-modified primers for detecting the copy number of SMN1 exon 7. The approach is suitable for newborn screening with trace DNA samples, such as DBS, and for carrier screening of pregnant women using peripheral blood DNA.

\section{ACKNOWLEDGEMENTS}

The authors greatly appreciate the support and corporation of all patients and their parents.

\section{AUTHOR CONTRIBUTIONS}

JP, ZL, and LW conceived and designed the study; JP, CZ, YT, SZ, and SC performed the experiments and analyzed the data; JP drafted the manuscript; and ZL, DL, and LW provided critical editing. All authors have read and agreed to the published version of the manuscript.

\section{CONFLICTS OF INTEREST}

No potential conflicts of interest relevant to this paper were reported.

\section{RESEARCH FUNDING}

This work was supported by grants from the National Key R\&D Program of China (2017YFC1001802, 2018YFC1002201), the Major Scientific and Technological Projects for Collaborative Pre- 
vention and Control of Birth Defects in Hunan Province (2019SK1010), and the National Natural Science Foundation of China (81771599).

\section{ORCID}

Jianyan Pan
Chunhua Zhang
Yanling Teng
Sijing Zeng
Siyi Chen
Desheng Liang
Zhuo Li
Lingqian Wu

https://orcid.org/0000-0002-0696-8261 https://orcid.org/0000-0001-8911-7843 https://orcid.org/0000-0002-1327-150X https://orcid.org/0000-0002-9165-2834 https://orcid.org/0000-0003-3918-5842 https://orcid.org/0000-0002-9451-8585 https://orcid.org/0000-0001-6010-6139 https://orcid.org/0000-0002-7070-5896

\section{REFERENCES}

1. Vill K, Kölbel H, Schwartz O, Blaschek A, Olgemöller B, Harms E, et al. One year of newborn screening for SMA-results of a German pilot project. J Neuromuscul Dis 2019;6:503-15.

2. Hendrickson BC, Donohoe C, Akmaev VR, Sugarman EA, Labrousse P, Boguslavskiy $L$, et al. Differences in SMN1 allele frequencies among ethnic groups within North America. J Med Genet 2009;46:641-4.

3 Sugarman EA, Nagan N, Zhu H, Akmaev VR, Zhou Z, Rohlfs EM, et al. Pan-ethnic carrier screening and prenatal diagnosis for spinal muscular atrophy: clinical laboratory analysis of $>72,400$ specimens. Eur J Hum Genet 2012;20:27-32.

4. Monaghan KG, Feldman GL, Palomaki GE, Spector EB, Ashkenazi Jewish Reproductive Screening Working Group, Molecular Subcommittee of the ACMG Laboratory Quality Assurance Committee. Technical standards and guidelines for reproductive screening in the Ashkenazi Jewish population. Genet Med 2008;10:57-72.

5. Grody WW, Thompson BH, Gregg AR, Bean LH, Monaghan KG, Schneider A, et al. ACMG position statement on prenatal/preconception expanded carrier screening. Genet Med 2013;15:482-3.

6. Lefebvre S, Bürglen L, Reboullet S, Clermont O, Burlet P, Viollet L, et al. Identification and characterization of a spinal muscular atrophy-determining gene. Cell 1995;80:155-65.

7. Bürglen $L$, Lefebvre $S$, Clermont $O$, Burlet $P$, Viollet $L$, Cruaud $C$, et al. Structure and organization of the human survival motor neurone (SMN) gene. Genomics 1996;32:479-82.

8. Chan V, Yip B, Yam I, Au P, Lin CK, Wong V, et al. Carrier incidence for spinal muscular atrophy in southern Chinese. J Neurol 2004;251:108993.

9. Su YN, Hung CC, Lin SY, Chen FY, Chern JP, Tsai C, et al. Carrier screening for spinal muscular atrophy (SMA) in 107,611 pregnant women during the period 2005-2009: a prospective population-based cohort study. PLoS One 2011;6:e17067.

10. Wang KC, Chang CC, Chang YF, Wang SH, Chiang CK, Tsai CP. Evaluation and characterization of a high-resolution melting analysis kit for rapid carrier-screening test of spinal muscular atrophy. J Neurogenet 2015;29:113-6.
11. Lundin KE, Højland T, Hansen BR, Persson R, Bramsen JB, Kjems J, et al. Biological activity and biotechnological aspects of locked nucleic acids. Adv Genet 2013;82:47-107.

12. Ishige T, Itoga S, Matsushita K. Locked nucleic acid technology for highly sensitive detection of somatic mutations in cancer. Adv Clin Chem 2018;83:53-72.

13. Edwards JG, Feldman G, Goldberg J, Gregg AR, Norton ME, Rose NC, et al. Expanded carrier screening in reproductive medicine-points to consider: a joint statement of the American College of Medical Genetics and Genomics, American College of Obstetricians and Gynecologists, National Society of Genetic Counselors, Perinatal Quality Foundation, and Society for Maternal-Fetal Medicine. Obstet Gynecol 2015;125: 653-62.

14 American College of Obstetricians and Gynecologists. Committee opinion No. 690 summary: carrier screening in the age of genomic medicine. Obstet Gynecol 2017;129: e35-e40.

15. Sumner CJ and Crawford TO. Two breakthrough gene-targeted treatments for spinal muscular atrophy: challenges remain. J Clin Invest 2018;128:3219-27.

16. Vidal-Folch N, Gavrilov D, Raymond K, Rinaldo P, Tortorelli S, Matern D, et al. Multiplex droplet digital PCR method applicable to newborn screening, carrier status, and assessment of spinal muscular atrophy. Clin Chem 2018;64:1753-61.

17. Feng Y, Ge X, Meng L, Scull J, Li J, Tian X, et al. The next generation of population-based spinal muscular atrophy carrier screening: comprehensive pan-ethnic SMN1 copy-number and sequence variant analysis by massively parallel sequencing. Genet Med 2017;19:936-44.

18. Mishra S, Lee Y, Park JW. Direct quantification of trace amounts of a chronic myeloid leukemia biomarker using locked nucleic acid capture probes. Anal Chem 2018;90:12824-31.

19. Ishige T, Satoh M, Itoga S, Nishimura M, Matsushita K, Nomura F. Highthroughput genotyping of GC (vitamin D-binding protein) by melting analysis with locked nucleic acid-incorporating dual hybridization probe for improving mismatch discrimination. Clin Chim Acta 2018;487:12632.

20. Taylor JL, Lee FK, Yazdanpanah GK, Staropoli JF, Liu M, Carulli JP, et al. Newborn blood spot screening test using multiplexed real-time PCR to simultaneously screen for spinal muscular atrophy and severe combined immunodeficiency. Clin Chem 2015;61:412-9.

21. Chien YH, Chiang SC, Weng WC, Lee NC, Lin CJ, Hsieh WS, et al. Presymptomatic diagnosis of spinal muscular atrophy through newborn screening. J Pediatr 2017;190:124-9.e1.

22. Gong B, Zhang L, Hou YP, Hu HY, Li HC, Tan MY, et al. Carrier screening for spinal muscular atrophy in 4719 pregnant women in Shanghai region [Article in Chinese]. Zhonghua Yi Xue Yi Chuan Xue Za Zhi 2013; 30:670-2.

23. Park JE, Yun SA, Roh EY, Yoon JH, Shin S, Ki CS. Carrier frequency of spinal muscular atrophy in a large-scale Korean population. Ann Lab Med 2020;40:326-30.

24. Mailman MD, Hemingway T, Darsey RL, Glasure CE, Huang Y, Chadwick RB, et al. Hybrids monosomal for human chromosome 5 reveal the presence of a spinal muscular atrophy (SMA) carrier with two SMN1 copies on one chromosome. Hum Genet 2001;108:109-15.

25. Wei X, Tan H, Yang P, Zhang R, Tan B, Zhang Y, et al. Notable carrier risks for individuals having two copies of SMN1 in spinal muscular atrophy families with 2-copy alleles: estimation based on Chinese meta-analysis data. J Genet Couns 2017;26:72-8. 
Supplemental Data Table S1. Primers and probes

\begin{tabular}{ll}
\hline Gene/primer/probe & \multicolumn{1}{c}{ Sequence } \\
\hline SMN1 & \\
Forward primer (SMN1-F)* & 5'-ATAAAGCTATCTATATATAGCTATCTAT G-3' \\
Reverse primer (SMN1-R) & 5'-CCTTCTTTTGATTTGTCT G-3' \\
Probe (SMN-P) & 5'-FAM-ACCCTGTAAGGAAAATAAAGGAAGT-MGB-3' \\
SMN2 & \\
Block probe (SMN2-B) & 5'-TCTTTTGATTTGTCT AAAACCC-C3 spacer-3' \\
ALB & \\
Forward primer (ALB-F) & 5'-CCCAGGTCAAGCCATTCTC-3' \\
Reverse primer (ALB-R) & 5'-GACTCTGTCACTACTGGCGTT-3' \\
Probe (ALB-P) & 5'-CY5-CCTGTCTTAGCTATCCGTG-MGB-3' \\
\hline
\end{tabular}

*Bases in bold and italics were LNA-modified, and the underlined bases indicate the specific site (c.835-44g/a and c.840C/T) of SMN1 and SMN2. Abbreviations: SMN, survival motor neuron; $A L B$, albumin; LNA, locked nucleic acid; MGB, minor groove binder; C3 spacer, three-carbon spacer; Cy5, cyanine 5. 
Pan J, et al.

Detecting SMA by qPCR with LNA-modified primers
ANNALS OF

LABORATORY MEDICINE

CAACTTAATTTCTGATCATATTTTGTTGAATAAAATAAGTAAAATGTCTTGTGAAACAAAATGCTTTTTAACATC CATATAAAGCTATCTATATATAGCTATCTATGTCTATATAGCTATTTTTTTTAACTTCCTTTATTTTCCTTACAGG GTTTCAGACAAAATCAAAAAGAAGGAAGGTGCTCACATTCCTTAAATTAAGGAGTAAGTCTGCCAGCATTATG AAAGTGAATCTTACTTTTGTAAAACTTTATGGTTTGTGGAAAACAAATGTTTTTGAACATTTAAAAAGTTCAGA TGTTAAAAAGTTGAAAGGTTAATGTAAAACAATCAATATTAAAGAATTTTGATGCCAAAACTATTAGATAAAA GGTTAATCTACATCCCTACTAGAATTCTCATACTTAACTGGTTGGTTATGTGGAAGAAACATACTTTCACAATA AAGAGCTTTAGGATATGATGCCATTTTATATCACTAGTAGGCAGACCAGCAGACTTTTTTTTATTGTGATATGG GATAACCTAGGCATACTGCACTGTACACTCTGACATATGAAGTGCTCTAGTCAAGTTTAACTGGTGTCCACAGA GGACATGGTTTAACTGGAATTCGTCAAGCCTCTGGTTCTAATTTCTCATTTGCAGGAAATGCTGGCATAGAGCA GCACTAAATGACACCACTAAAGAAACGATCAGACAGATCTGGAATGTGAAGCGTTATAGAAGATAACTGGCC TCATTTCTTCAAAATATCAAGTGTTGGGAAAGAAAAAAGGAAGTGGAATGGGTAACTCTTCTTGATTAAAAGT TATGTAATAACCAAATGCAATGTGAAATATTTTACTGGACTCTATTTTGAAAAACCATCTGTAAAAGACTGGGG TGGGGGTGGGAGGCCAGCACGGTGGTGAGGCAGTTGAGAAAATTTGAATGTGGATTAGATTTTGAATGATA TTGGATAATTATTGGTAATTTTATGAGCTGTGAGAAGGGTGTTGTAGTTTATAAAAGACTGTCTTAATTTGCAT ACTTAAGCATTTAGGAATGAAGTGTTAGAGTGTCTTAAAATGTTTCAAATGGTTTAACAAAATGTATGTGAGG CGTATGTGGCAAAATGTTACAGAATCTAACTGGTGGACATGGCTGTTCATTGTACTGTTTTTTTCTATCTTCTAT ATGTTTAAAAGTATATAATAAAAATATTTAATTTTTTTTTTAAATTAGCTGTATCTGTGATTGTATTTCTTTTAGAG TCTTATCTATGAAGGTTAAAAACAAGAAGAGACATATTATACAGTAGATATTTATTGTGTGGCTCATACACATG GTGCTCTTCTGATTATGGATTTTAGAGATAATAACAGTGAACAAGACATAGTTTCTTTTCCTCGAGTAGATTAAA GTCATACATTGACTTTTAATGGTGACTGGCATTCTTAATACATGATTATTATATATTAGGTACCATGTCAGATTA ATTATAATACTTTTACTACTTTTTAATTTAACCCTTGAACTATCCCTATTGAGTCAGATATATTTCCTTCCATTTTCTA CTTGTATCTTTCAAGTTTAGCATATGCTGATACATATGAAGCTCTCTCCAGGTTTTATTGAAAGAAGAAATTAAT AAATTTATTAATGTCACTGAATTAGGCAACTCACTTTCCCAAGATTATGCAAGTGGTACAGGTGGAACTCAAA GCCAAGTTTAACTAGTTGTTCAGGAGAATGTTTTCTACCCTCCACTAACCCACTACTCTGCAGATGGAGATAAT ATGATGAATGGAACATAGCAACATCTTAGTTGATTCCGGCCAAGTGTTCTCTGTTTTATCTACTATGTTAGACA GTTTCTTGCCTTGCTGAAAACACATGACTTCTTTTTTTTCAGGCTATTAGTTCGTTACACCAAGAAAGTACCCCAA GTGTCAACTCCAACTCTTGTAGAGGTCTCAAGAAACCTAGGAAAAGTGGGCAGCAAATGTTGTAAACATCCTG AAGCAAAAAGAATGCCCTGTGCAGAAGACTATGTGAGTCTTTAAAAAAATATAATAAATTAATAATGAAAAAA TTTTACCTTTAGATATTGATAATGCTAGCTTTCATAAGCAGAAGGAAGTAATGTGTGTGTGTGCATGTTTGTGT GCATGTGTGTGTGCATGCACGTGTGTGTATGTGTGATATTGGCAGTCAAGGCCCCGAGGATGATAATTTTTTT TTTTTTTTTGAGACGGAGTCTCGCTTTGTTGTCCAGGCTGGAGTGCAGTGGTGCCATCTCGGCTCACTGCAACC TCCGCCTCCCAGGTTCAAGCCATTCTCCTGCCTCAGCCTCCCAAGTAGCTGGGACTACAGGTGCATGCCACCAT GCCTGGCTAATTTTTTGTATTTTTAGTAGAAAATTTTCAGCTTCACCTCTTTTGAATTTCTGCTCTCCTGCCTGTT CTTTAGCTATCCGTGGTCCTGAACCAGTTATGTGTGTTGCATGAGAAAACGCCAGTAAGTGACAGAGTCACCA AATGCTGCACAGAATCCTTGGTGAACAGGCGACCATGCTTTTCAGCTCTGGAAGTCGATGAAACATACGTTCC CAAAGAGTTTA

Supplemental Data Fig. S1. The SMN1 (blue) and ALB (red) sequences that were cloned into a single pUC57 vector as the standard samples.

Abbreviations: SMN1, survival motor neuron 1; $A L B$, albumin. 
SMN-F/SMN-R

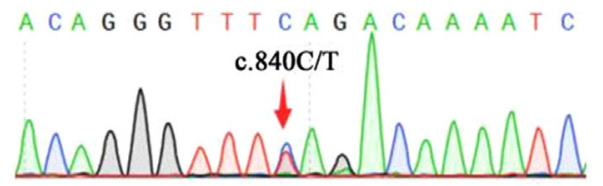

SMNI-F1/SMN-R

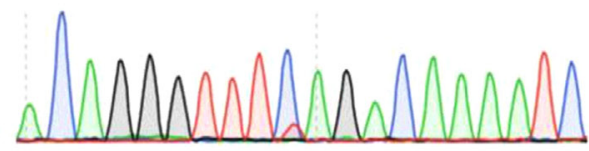

G C T A T C T A T G T C TATATA G C c. $835-44 \mathrm{~g} / \mathrm{a}$

SMN-F/SMN-R

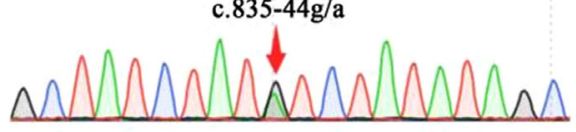

SMN-F/SMN1-R1

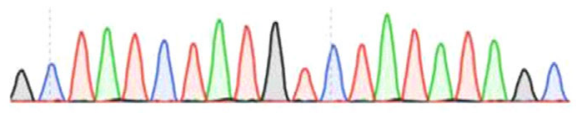

\section{SMN1-F/SMN-R}

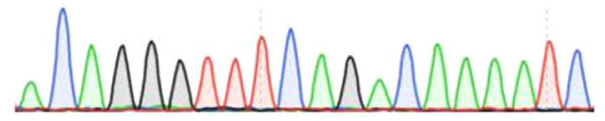

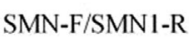

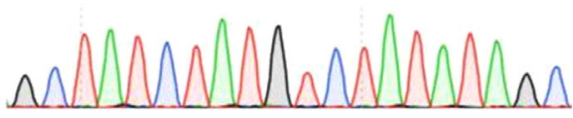

Supplemental Data Fig. S2. The specificity of SMN1-F and SMN1-R was confirmed by Sanger sequencing. (A) The height of the C.840C/ T sequencing peak was used to verify the specificity of primers SMN1-F1 and SMN1-F. The PCR products of non-specific primers SMN-F and SMN-R exhibited nearly equal height C/T peaks. A low T peak appeared in the PCR product of non-specific primer SMN-R and specific primer SMN1-F1 (without LNA modification). A single C peak appeared in the PCR product of non-specific primer SMN-R and specific primer SMN1-F (with LNA modification), indicating complete elimination of nonspecific amplification. (B) The height of the c.835-44g/a sequencing peak was used to verify the specificity of primers SMN1-R1 and SMN1-R. The PCR products of non-Specific primers SMN-F and SMN-R exhibited nearly equal height g/a peaks. A single g peak appeared in the PCR products of non-specific primer SMN-F and specific primer SMN1-R1 or SMN1-R (without or with LNA modification), indicating that both specific reverse primers exhibit high specificity for SMN1.

Abbreviations: SMN1, survival motor neuron 1; LNA, locked nucleic acid; F, forward; R, reverse. 